\title{
Erratum: Catalytic Quantum Randomness [Phys. Rev. X 8, 041016 (2018)]
}

\author{
P. Boes, H. Wilming, R. Gallego, and J. Eisert
}

(Received 5 March 2019; accepted 13 December 2019; published 15 April 2020)

DOI: 10.1103/PhysRevX.10.029901

We report an error found in this Letter: In Sec. V, there is both a typographic error in Theorem 3 as well as a false statement concerning the speed of convergence of the expander graph construction that we present in the section. The relevant scaling is exponential with the system size (i.e., the logarithm of the Hilbert-space dimension) rather than with the Hilbert-space dimension of the source of randomness $R$, as originally claimed. There are corresponding typographic errors in the statement of Theorem 3, in which every occurrence of the term $8 k$ should be corrected to $8^{k}$. Furthermore, in the statement of the theorem, it should read $U \in \mathrm{U}\left(d 8^{k}\right)$ instead of $U \in(d 8 k), \mathrm{U}(n)$ denoting the group of $n \times n$ unitary matrices. Given these corrections to the statement of the theorem, the theorem is correct and so is the proof.

Published by the American Physical Society under the terms of the Creative Commons Attribution 4.0 International license. Further distribution of this work must maintain attribution to the author(s) and the published articles title, journal citation, and DOI. 